\title{
Identification of the optimal wavelengths for optical topography: a photon measurement density function analysis
}

\author{
Teresa Correia \\ Adam Gibson \\ Jeremy Hebden \\ University College London \\ Department of Medical Physics and Bioengineering \\ Gower Street \\ London, WC1E 6BT United Kingdom
}

\begin{abstract}
A method is presented to select the optimal wavelengths for multispectral optical topography, which not only gives good separation between chromophores, absorption, and scattering, but also minimizes the differences between interrogated volumes. This method uses the sum of squared differences to compare photon measurement density functions, which were generated for wavelengths in the nearinfrared (NIR) range for a suitable model of tissue optical properties. It is found that including this condition significantly influences the range of optimal wavelengths. However, for the adult human head, the differences between interrogated volumes at NIR wavelengths are very small and image reconstruction is only slightly improved when measurements with overlapping sensitivities are used. () 2010 Society of PhotoOptical Instrumentation Engineers. [DOI: 10.1117/1.3484747]
\end{abstract}

Keywords: optical topography; multispectral reconstruction; optimal wavelengths; photon measurement density function.

Paper 10169PR received Apr. 7, 2010; revised manuscript received Jul. 13, 2010; accepted for publication Jul. 16, 2010; published online Sep. 16, 2010.

\section{Introduction}

Diffuse optical imaging is a noninvasive technique used to obtain the spatial distribution of optical properties and chromophore concentrations of tissue from measurements of nearinfrared (NIR) light, which propagates through the tissue. The technique can be used to monitor cerebral oxygenation and hemodynamics, to provide information about cerebral pathological conditions, to measure functional brain activation during a physical or mental task, and for breast cancer detection. ${ }^{1-6}$ Two different approaches are considered in NIR imaging: optical topography and optical tomography. Optical topography is used to describe systems that obtain diffuse reflectance measurements from an array of source-detector pairs placed on the surface of the tissue, whereas optical tomography involves reconstructing a 3-D volume using multiple measurement of transmitted light across the whole tissue volume. $^{7}$

A few NIR spectroscopy studies have been published that explore the selection of an optimal wavelength pair that minimizes cross-talk, where a change in oxy-hemoglobin concentration $\left[\mathrm{HbO}_{2}\right]$ may replicate a change in deoxy-hemoglobin concentration $[\mathrm{HHb}]$ and vice versa, and minimizes the influence of noise on calculated chromophore concentration changes. ${ }^{8-13}$ These studies revealed that, for measurements performed at two different NIR wavelengths, one of the wavelengths should be in the range $650-750 \mathrm{~nm}$ and the other $>820 \mathrm{~nm}$.

Address all correspondence to: Teresa Correia, University College London, Department of Medical Physics and Bioengineering, Gower Street, London, WC1E 6BT, United Kingdom. Tel: 44-20-7679-0264; Fax: 44-20-7679-0255. E-mail: tcorreia@medphys.ucl.ac.uk
Image reconstruction using continuous wave $(\mathrm{CW})$ measurements at a single wavelength is an inverse problem with nonunique solutions, where multiple sets of optical properties can yield similar data. ${ }^{14}$ As a consequence, cross-talk between the images of absorption and scattering occurs. Increasing the number of measurement wavelengths only increases the number of unknowns because absorption and scattering need to be reconstructed for each wavelength. However, the multispectral method proposed by Corlu et al. ${ }^{15,16}$ directly reconstructs the chromophore concentrations and scattering factors that are wavelength independent, meaning that the number of unknowns remains constant when the number of measurement wavelengths increases, representing a clear advantage over the conventional method.

Corlu et al. ${ }^{15,16}$ introduced an approach for selecting the optimal wavelengths for optical tomography based on their multispectral method. This approach sought to maximize separation between absorption and scattering separation while also optimizing separation between the concentrations of main breast tissue chromophores $\left(\mathrm{HbO}_{2}, \mathrm{HHb}\right.$, water, and lipid). However, this overlooks the possibility that the volumes over which measurements are sensitive to changes in absorption and scattering may be different at the selected wavelengths. This issue is of importance in order to accurately locate optical property changes occurring within the object of study, because different wavelengths may have different sensitivities to the same changes in the medium, and the same measured change could be associated with changes in different regions within the reconstructed volume. In this study, the optimal wavelength set is estimated by incorporating a third condition, which takes into account the interro-

1083-3668/2010/15(5)/056002/14/\$25.00 @ 2010 SPIE 
gated volume. The spatial similarities between sensitivity profiles for different wavelengths are analyzed and quantified.

\section{Theory}

\subsection{Reconstruction Method}

The conventional image reconstruction method involves finding the optical properties of the medium from a set of measurements made at the surface. The optical properties are then combined to calculate the chromophore concentrations at wavelength $\lambda$ using the following equation:

$$
\mu_{a}(\lambda)=\Sigma_{i} \varepsilon_{i}(\lambda) c_{i},
$$

where $\varepsilon_{i}$ is the extinction coefficient and $c_{i}$ is the concentration of the $i$ 'th chromophore. The reduced scattering coefficient $\mu_{\mathrm{s}}^{\prime}$ is approximately given by Mie scattering theory as

$$
\mu_{\mathrm{s}}^{\prime}(\lambda)=a \lambda^{-b},
$$

where $a$ is the scatter amplitude and $b$ the scatter power. In this work, the latter is considered to be constant for simplicity.

The multispectral method involves reconstructing chromophore concentrations and scatter parameters using all measurements at all wavelengths simultaneously. ${ }^{16}$ The problem is nonlinear but can be linearized and reduced to the so-called difference method, based on the assumption that the true chromophore concentrations are close to an initial guess. For CW measurements, only intensity $A$ is measured, hence changes in the measured data $\Delta A$ at $n$ measurement wavelengths are related to changes in the chromophore concentrations $\Delta c_{i}$ and scatter amplitude $\Delta a$ through matrix $\widetilde{J}$,

$$
\begin{aligned}
\left(\begin{array}{c}
\Delta A_{\lambda_{1}} \\
\Delta A_{\lambda_{2}} \\
\ldots \\
\Delta A_{\lambda_{n}}
\end{array}\right)= & \left(\begin{array}{ccccc}
\widetilde{J}\left(c_{1}, \lambda_{1}\right) & \widetilde{J}\left(c_{2}, \lambda_{1}\right) & \ldots & \widetilde{J}\left(c_{i}, \lambda_{1}\right) & \widetilde{J}\left(a, \lambda_{1}\right) \\
\widetilde{J}\left(c_{1}, \lambda_{2}\right) & \widetilde{J}\left(c_{2}, \lambda_{2}\right) & \ldots & \widetilde{J}\left(c_{i}, \lambda_{2}\right) & \widetilde{J}\left(a, \lambda_{2}\right) \\
\vdots & \vdots & \vdots & \vdots & \vdots \\
\widetilde{J}\left(c_{1}, \lambda_{n}\right) & \widetilde{J}\left(c_{2}, \lambda_{n}\right) & \ldots & \widetilde{J}\left(c_{i}, \lambda_{n}\right) & \widetilde{J}\left(a, \lambda_{n}\right)
\end{array}\right) \\
& \times\left(\begin{array}{c}
\Delta c_{1} \\
\Delta c_{2} \\
\ldots \\
\Delta c_{i} \\
\Delta a
\end{array}\right),
\end{aligned}
$$

where the elements of the matrix $\widetilde{J}$ are given by

$$
\begin{aligned}
\widetilde{J}\left(c_{i}, \lambda\right) & =\frac{\partial A(\lambda)}{\partial c_{i}}=\frac{\partial A(\lambda)}{\partial \mu_{a}(\lambda)} \frac{\partial \mu_{a}(\lambda)}{\partial c_{i}}=\frac{\partial A(\lambda)}{\partial \mu_{a}(\lambda)} \varepsilon_{i}(\lambda) \\
& =J_{\lambda}\left(\mu_{a}\right) \varepsilon_{i}(\lambda),
\end{aligned}
$$

and

$$
\begin{aligned}
\widetilde{J}(a, \lambda) & =\frac{\partial A(\lambda)}{\partial a}=\frac{\partial A(\lambda)}{\partial \kappa(\lambda)} \frac{\partial \kappa(\lambda)}{\partial a}=\frac{\partial A(\lambda)}{\partial \kappa(\lambda)} \frac{\partial \kappa(\lambda)}{\partial \mu_{\mathrm{s}}^{\prime}(\lambda)} \frac{\partial \mu_{\mathrm{s}}^{\prime}(\lambda)}{\partial a} \\
& =J_{\lambda}(\kappa)\left[-3 \kappa(\lambda)^{2}\right]\left(\lambda^{-b}\right) .
\end{aligned}
$$

where $\kappa$ is the diffusion coefficient. The Jacobian matrix $J_{\lambda}$ represents the sensitivity of a measurement to changes in the optical properties within the medium. The Jacobian can be calculated by solving the diffusion equation using the finite element method, for both the forward and adjoint solutions. ${ }^{17}$ In the Rytov approximation, changes in the log intensity are used instead. ${ }^{17,18}$

Chromophore concentrations and scatter parameters can be obtained by solving an ill-posed inverse problem at all wavelengths. In the work presented here, this is aided using the zero-order Tikhonov regularization,

$$
\left[\Delta c_{1}, \cdots, \Delta c_{i}, \Delta a\right]^{T}=\left(\tilde{J}^{T} \widetilde{J}+\alpha I\right)^{-1} \widetilde{J}^{T}\left[\Delta A_{\lambda_{1}}, \ldots, \Delta A_{\lambda_{n}}\right]^{T},
$$

where $\alpha=\operatorname{diag}\left(J^{T} J+\gamma\right)^{1 / 2}$ is the NOSER-type regularization parameter, ${ }^{19,20}$ with $\gamma=\beta \sigma_{\max }^{-1}$, where $\beta$ is a scaling factor and $\sigma_{\max }$ is the maximum singular value of $J^{T} J$. The constant $\gamma$ ensures that the regularization parameter is never too small and is approximately equal to the noise present in the data.

\subsection{Identification of the Optimal Wavelengths in Optical Topography}

\subsubsection{Unique and simultaneous reconstruction}

The nonuniqueness problem associated with using CW measurements results in cross-talk between absorption and scattering. Minimizing the nonuniqueness of the multispectral method by selecting the optimal wavelengths represents one of the conditions introduced by Corlu et al. ${ }^{16}$ Rewriting the nonuniqueness conditions introduced by Arridge and Lionheart ${ }^{14}$ in terms of the wavelength-independent variables in the multispectral method, it follows that the inverse problem has a nonunique solution when the residual norm ${ }^{16} R$ :

$$
R=\left\|1-E\left(E^{T} E\right)^{-1} E^{T} 1\right\|
$$

is zero. Thus, a maximization of $R$ is required. $E$ is a wavelength-dependent matrix of the form

$$
E(\lambda)=\left(\begin{array}{cccc}
\frac{\varepsilon_{1}\left(\lambda_{1}\right)}{\lambda_{1}^{b}} & \frac{\varepsilon_{2}\left(\lambda_{1}\right)}{\lambda_{1}^{b}} & \ldots & \frac{\varepsilon_{i}\left(\lambda_{1}\right)}{\lambda_{1}^{b}} \\
\vdots & \vdots & \vdots & \vdots \\
\frac{\varepsilon_{1}\left(\lambda_{n}\right)}{\lambda_{n}^{b}} & \frac{\varepsilon_{2}\left(\lambda_{n}\right)}{\lambda_{n}^{b}} & \cdots & \frac{\varepsilon_{i}\left(\lambda_{n}\right)}{\lambda_{n}^{b}}
\end{array}\right) .
$$

The ability to separate chromophore concentrations can be evaluated from the matrix form of Eq. (1), ${ }^{16}$

$$
\left(\begin{array}{c}
\Delta \mu_{a} \lambda_{1} \\
\Delta \mu_{a} \lambda_{2} \\
\cdots \\
\Delta \mu_{a} \lambda_{n}
\end{array}\right)=\left(\begin{array}{cccc}
\varepsilon_{1}\left(\lambda_{1}\right) & \varepsilon_{2}\left(\lambda_{1}\right) & \cdots & \varepsilon_{i}\left(\lambda_{1}\right) \\
\vdots & \vdots & \vdots & \vdots \\
\varepsilon_{1}\left(\lambda_{n}\right) & \varepsilon_{2}\left(\lambda_{n}\right) & \cdots & \varepsilon_{i}\left(\lambda_{n}\right)
\end{array}\right)\left(\begin{array}{c}
\Delta c_{1} \\
\Delta c_{2} \\
\cdots \\
\Delta c_{i}
\end{array}\right)
$$

using the condition number $\mathbf{k}$ of the extinction coefficient matrix $\varepsilon_{i}(\lambda)$, which is the ratio of the largest to the smallest singular value of the matrix. A small $\mathbf{k}\left[\varepsilon_{i}(\lambda)\right]$ indicates a smooth singular value decay resulting in a reduced sensitivity of the system to errors in the measurements, and therefore, chromophores contribute evenly to absorption. In order to distinguish between chromophore concentrations, the wave- 
length sets chosen should minimize the condition number $\mathbf{k}\left[\varepsilon_{i}(\lambda)\right]$, which defines the second condition introduced by Corlu et al. ${ }^{16}$ This condition number also describes the linear independence of the system of equations. When $\mathbf{k}\left[\varepsilon_{i}(\lambda)\right]$ is small, the matrix has full rank and, therefore, the wavelength set provides distinct and independent information.

\subsubsection{Interrogated volume}

In optical topography brain activation studies using multiple wavelengths, it is invariably assumed that measurements at each wavelength contain information from the same cerebral region. If not, data at some wavelength could be measuring a region where the activation occurs, whereas the data at others could be more sensitive to surrounding areas and the study may return erroneous results. Each row of the Jacobian matrix $J$ represents the measurement sensitivity of a source-detector pair to a perturbation in the optical properties of the object of study, which is known as the photon measurement density function (PMDF). ${ }^{21}$ In terms of image reconstruction, if the forward model is calculated for the same optical properties but the measurement wavelengths have different sensitivities to changes in absorption and scattering, then for a perturbation in the optical properties of the tissue, the reconstructed perturbation will occur in different spatial locations. In practice, the optical properties of tissues beneath the probe are unknown, and in order to reconstruct changes in tissue properties as accurately as possible, it is necessary to acquire data at wavelengths where the sensitivities at each wavelength coincide.

In this work, a method is proposed to find which combinations of wavelengths have overlapping sensitivities, which is an indicator of the similarity between interrogated volumes, by comparing PMDFs for a certain source-detector pair using different measurement wavelengths. The influence of using different NIR measurements is analyzed in terms of image reconstruction and volume sensitivities. This is less relevant when considering transmission measurements (e.g., across a head of breast) because there is an intrinsically greater overlap of the PMDFs.

\section{Methods}

The software package TOAST (time-resolved optical absorption and scattering tomography) $)^{22}$ was used to model the propagation of light in tissue at wavelengths from 650 to $990 \mathrm{~nm}$ spaced in $10-\mathrm{nm}$ intervals. To generate PMDFs for each wavelength, one needs to insert the corresponding optical properties of the medium in the forward problem. Initially, the medium is considered to be homogeneous with optical properties of gray matter. Subsequently, a three-layer model is used to generate PMDFs, which consists of two extracerebral layers with skin and skull optical properties and an intracerebral layer with gray-matter optical properties. A single source-detector pair $40 \mathrm{~mm}$ apart was used in the forward model because simulations have indicated that this is sufficient to measure hemodynamic changes in adult gray matter. $^{23}$

\subsection{Adult Head Model}

\subsubsection{Homogeneous model}

The first model consisted of a uniform slab with dimensions $85 \times 85 \times 30 \mathrm{~mm}$ with intracerebral tissue optical properties derived from a combination of chromophores present in brain tissue: hemoglobin, water, lipid, and cytochromes.

Brain absorption spectrum. Total absorption coefficient for brain tissue is calculated from Eq. (1) as

$$
\begin{aligned}
\mu_{a, \text { brain }}(\lambda)= & \varepsilon_{\mathrm{HbO}_{2}}[\mathrm{HbT}] \mathrm{SO}_{2}+\varepsilon_{\mathrm{HHb}}[\mathrm{HbT}]\left(1-\mathrm{SO}_{2}\right) \\
& +\mu_{a, \mathrm{H}_{2} \mathrm{O}} W+\mu_{a, \text { Lipid }} L \\
& +\sum_{a a 3, b, c}\left\{\varepsilon_{\text {oxCyt } x}[\text { oxCyt } x] \mathrm{Ox}\right. \\
& \left.+\varepsilon_{r \text { Cyt } x}[r \text { Cyt } x](1-\mathrm{Ox})\right\}+B,
\end{aligned}
$$

where $[\mathrm{HbT}]$ is the total hemoglobin concentration [in micromolar $(\mu \mathrm{M})], \mathrm{SO}_{2}$ is the oxygen saturation, $W$ is the fraction of water content, $\mu_{a, \mathrm{H}_{2} \mathrm{O}}$ is the absorption coefficient of $100 \%$ water $\left(\mathrm{mm}^{-1}\right), L$ is the fraction of lipid content, $\mu_{a \text {,Lipid }}$ is the absorption coefficient of $100 \%$ fat $\left(\mathrm{mm}^{-1}\right), \varepsilon_{\mathrm{HHb}}$ is the specific absorption coefficient of $\mathrm{HHb}\left(\mu \mathrm{M}^{-1} \mathrm{~mm}^{-1}\right), \varepsilon_{\mathrm{HbO}_{2}}$ is the specific absorption coefficient of $\mathrm{HbO}_{2}\left(\mu \mathrm{M}^{-1} \mathrm{~mm}^{-1}\right)$, $\varepsilon_{r \text { Cyt } x}$ is the specific absorption coefficient of reduced cytochrome $x\left(\mu \mathrm{M}^{-1} \mathrm{~mm}^{-1}\right), \varepsilon_{\text {oxCyt } x}$ is the specific absorption coefficient of oxidised cytochrome $x\left(\mu \mathrm{M}^{-1} \mathrm{~mm}^{-1}\right)$, and $\mathrm{Ox}$ is the fraction of oxidized Cyt $x$. The parameter $B$ is the background absorption, which was set to $B=0.012 \mathrm{~mm}^{-1}$ so that the total absorption matches the value $\mu_{a}(800 \mathrm{~nm})$ $=0.036 \mathrm{~mm}^{-1}$ used by Okada and Delpy. ${ }^{31}$ The extinction coefficients used in this work were obtained from the literature. ${ }^{26,32-34}$ The estimated concentrations in the gray matter for each chromophore are summarized in Table 1.

Brain scattering spectrum. Matcher et al. ${ }^{35}$ measured $\mu_{\mathrm{s}}^{\prime}$ of the forehead of different human adults over the wavelength range of 760-900 $\mathrm{nm}$. They fitted the experimental spectra to Eq. (2) and obtained $a=32.08\left(\mathrm{~mm}^{-1} \mathrm{~nm}^{-1}\right)$ and $b$ $=0.53\left(\mathrm{~mm}^{-1}\right)$. Meanwhile, van der Zee ${ }^{27}$ measured $\mu_{\mathrm{s}}^{\prime}$ for gray matter in vitro and found $\mu_{\mathrm{s}}^{\prime}(800 \mathrm{~nm})=2.2 \mathrm{~mm}^{-1}$, which can be used as a reference number and included in the exponential relation found by Matcher et al., ${ }^{35}$ which then becomes

$$
\mu_{\mathrm{s}^{\prime}, \text { brain }}(\lambda)=\frac{2.2\left(a \lambda^{-b}\right)}{\left(a 800^{-b}\right)} .
$$

\subsubsection{Three-layer model}

Figure 1 shows the second adult head model, which is a slab with the same dimensions as the homogeneous model, consisting of three layers that imitate the scalp, skull, and intracerebral tissue. Scalp is represented by a $4-\mathrm{mm}$ thickness layer, and the skull is $7 \mathrm{~mm}$ thick. Absorption and scattering spectra of skull are described in Firbank et al. ${ }^{36}$

Skin absorption spectrum. Three main chromophores were considered for scalp: hemoglobin, water, and melanin. Their 
Table 1 Chromophore concentrations used to calculate the absorption coefficients for the skin and gray matter.

\begin{tabular}{|c|c|c|}
\hline Layer & Parameter & \\
\hline \multirow[t]{13}{*}{ Gray matter } & Water content $(\%)^{24}$ & 80 \\
\hline & Lipid content $(\%)^{25}$ & 11.6 \\
\hline & {$[\mathrm{HbT}](\mu \mathrm{M})^{26}$} & 80 \\
\hline & $\mathrm{SO}_{2}(\%)^{26}$ & 70 \\
\hline & [oxCył aa3] $(\mu \mathrm{M})^{27}$ & 6.4 \\
\hline & {$\left[\mathrm{rCyt}\right.$ aa3] $(\mu \mathrm{M})^{27}$} & 1.6 \\
\hline & $\mathrm{O}_{a a 3}(\%)^{27}$ & 80 \\
\hline & {$[o x C y+b](\mu M)^{27}$} & 2.37 \\
\hline & {$[\mathrm{rCyt} b](\mu \mathrm{M})^{27}$} & 0.89 \\
\hline & $\mathrm{O}_{c}(\%)^{27}$ & 50 \\
\hline & {$[\mathrm{oxCyt} c](\mu \mathrm{M})^{27}$} & 1.36 \\
\hline & {$[\mathrm{rCyt} c](\mu \mathrm{M})^{27}$} & 0.68 \\
\hline & $\mathrm{O}_{b}{ }^{27}$ & 40 \\
\hline \multirow[t]{4}{*}{ Skin } & Water content $(\%)^{28}$ & 60 \\
\hline & {$[\mathrm{HbT}](\mu \mathrm{M})^{29}$} & 65 \\
\hline & $\mathrm{SO}_{2}(\%)^{28}$ & 71 \\
\hline & Melanin content $(\%)^{30}$ & 13 \\
\hline
\end{tabular}

estimated concentrations in skin are summarized in Table 1. The melanin absorption coefficient can be approximated by the expression ${ }^{30}$

$$
\mu_{a \text {, melanin }}=\left(6.6 \times 10^{10}\right) \lambda^{-3.33},
$$

in $\mathrm{mm}^{-1}$, where $\lambda$ is the wavelength represented in units of nanometers. Considering the contributions from all skin chromophores, the total absorption coefficient of the skin layer can be represented as

$$
\begin{aligned}
\mu_{a, \text { skin }}(\lambda)= & \epsilon_{\mathrm{HbO}_{2}}[\mathrm{HbT}] \mathrm{SO}_{2}+\epsilon_{\mathrm{HHb}}[\mathrm{HbT}]\left(1-\mathrm{SO}_{2}\right) \\
& +\mu_{a, \mathrm{H}_{2} \mathrm{O}} W+\mu_{a, \text { melanin }} M,
\end{aligned}
$$

where $M$ is the fraction of melanin content. It was not necessary to consider a background absorption for the skin layer, because the absorption coefficient values already show a good agreement with the values presented by Simpson et al. ${ }^{37}$

Skin scattering spectrum. The wavelength dependence of the reduced scattering coefficient of skin has been modeled by Bashkatov et al. ${ }^{38}$ as a combination of Mie and Rayleigh scattering given by

$$
\mu_{\mathrm{s}, \mathrm{skin}}^{\prime}=7.37 \lambda^{-0.22}+1.1 \times 10^{11} \lambda^{-4},
$$

in $\mathrm{mm}^{-1}$, where $\lambda$ is the wavelength in $\mathrm{nm}$. The first term corresponds to Mie scattering due to large collagen fibers and the second to Rayleigh scattering mainly due to small collagen fibrils.

\subsection{Similarity Measures}

The sum of squared differences (SSD) can be used to measure the similarity between two sensitivity profiles. For PMDFs $X$ and $Y$ with voxels $i$, the SSD is defined as

$$
\mathrm{SSD}=\frac{1}{N} \sum_{i}^{N}|X(i)-Y(i)|^{2} \quad \forall i \in X \cap Y,
$$

where $N$ is the number of voxels. The SSD will be zero when the distributions are identical.

\subsection{Optimal Wavelengths}

Sensitivity profile maps (PMDFs) were obtained for the wavelength range of $650-990 \mathrm{~nm}$ in steps of $10 \mathrm{~nm}$, making a total of 35 wavelengths. Initially, the PMDFs were combined in sets of three wavelengths $\left(C_{3}^{35}\right)$, making a total of 6545 wavelength sets. Thereafter, wavelength sets of four wavelengths were investigated, which results in 52,360 sets. For a medium with $i$ absorption chromophores, at least $n=i+1$ wavelengths are required when the scattering parameter $a$ is allowed to vary. ${ }^{16}$ Therefore, in order to find the solution for both absorption and scattering coefficients for a medium with two chromophores, it is necessary to employ at least three wavelengths. It is common for optical topography systems to acquire data at just two wavelengths. Therefore, all possible

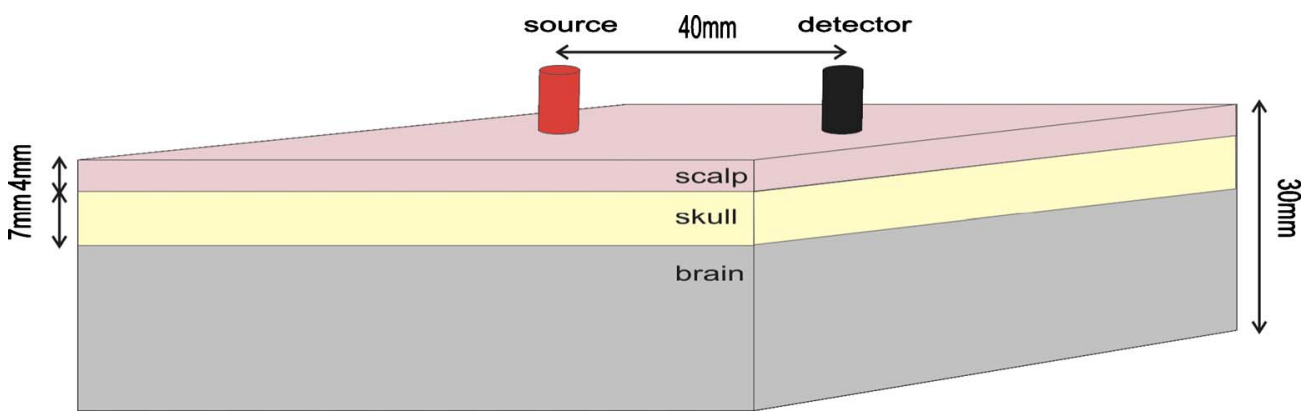

Fig. 1 Three-layer adult head model and probe placement. 
wavelength pairs are compared (total of 595), to provide an insight into what can be achieved using only two measurement wavelengths.

The aim of this study is to determine which wavelength set gives the best combination of: (i) good separation between absorption and scattering, (ii) good separation between $\left[\mathrm{HbO}_{2}\right]$ and $[\mathrm{HHb}]$, and (iii) excellent overlap between sensitivity profiles. Quantitatively, we aim to find the wavelength sets that maximize the residual norm $R$ [Eq. (7)], minimize the condition number $\mathbf{k}$ of the matrix $\varepsilon_{i}(\lambda)$ [Eq. (9)], and minimize the SSD [Eq. (15)]. The matrix $E$ in Eq. (8) for three wavelengths is reduced to

$$
E(\lambda)=\left(\begin{array}{cc}
\frac{\varepsilon_{\mathrm{HbO}_{2}}\left(\lambda_{1}\right)}{\lambda_{1}^{b}} & \frac{\varepsilon_{\mathrm{Hbb}}\left(\lambda_{1}\right)}{\lambda_{1}^{b}} \\
\frac{\varepsilon_{\mathrm{HbO}_{2}}\left(\lambda_{2}\right)}{\lambda_{2}^{b}} & \frac{\varepsilon_{\mathrm{Hbb}}\left(\lambda_{2}\right)}{\lambda_{2}^{b}} \\
\frac{\varepsilon_{\mathrm{HbO}_{2}}\left(\lambda_{3}\right)}{\lambda_{3}^{b}} & \frac{\varepsilon_{\mathrm{Hbb}}\left(\lambda_{3}\right)}{\lambda_{3}^{b}}
\end{array}\right),
$$

where the scatter power $b$ is considered to be $0.53 .{ }^{35}$

\subsection{PMDF Comparison}

The PMDFs for wavelengths from 650 to $990 \mathrm{~nm}$ are compared by quantifying the differences in their sensitivity in terms of penetration depth, volume, and full width at half maximum (FWHM), in order to determine if these differences are significant. Absolute sensitivity values are used (because the absorption PMDF has negative sensitivity), and the threshold is set to 0.01 . An analysis is performed of how these differences affect the measurements and image reconstructions.

\subsubsection{Volume}

The volume $(V)$ is the number of voxels $(N)$ with sensitivity above the threshold times the voxel volume. The relative volume compares the PMDF volume at $650 \mathrm{~nm}\left(V_{650}\right)$ with all the other PMDF volumes $\left(V_{\lambda}\right)$,

$$
\text { relative volume }=\frac{V_{\lambda}}{V_{650}}=\frac{N_{\lambda}}{N_{650}},
$$

where $N_{650}$ is the number of voxels above the threshold in the PMDF at $650 \mathrm{~nm}$ and $N_{\lambda}$ is the number of voxels above the threshold for the remaining wavelengths.

\subsection{2 $F W H M$}

The FWHM was calculated for the PMDF cross section at half-way between the source and detector for the depth direction. The PMDF profiles include the point of maximum sensitivity. The FWHM provides a measure of the spread of the PMDFs.

\subsubsection{Penetration depth}

The penetration depth (PD) is defined as the maximum distance into the medium at which perturbations in the optical properties can still be measured. The central region of the PMDF penetrates deeper into the tissue, which corresponds to the PMDF profile ( $z$ direction) that passes through the mid-

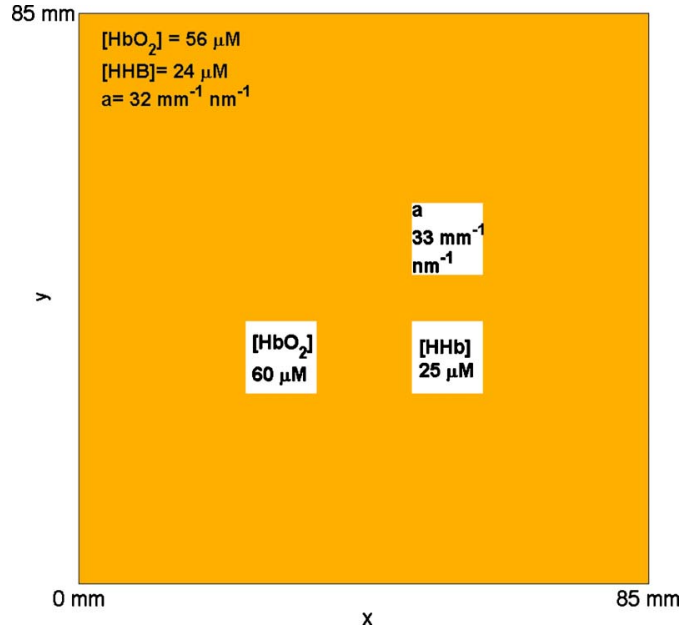

Fig. 2 Cross section $x y$ at $z=11.7 \mathrm{~mm}$ of the medium used in the simulation.

point between the source and detector. Hence, the distance at which the sensitivity drops below the threshold for these profiles is considered to be the PD.

\subsection{Image Reconstruction}

Simulations were performed to compare the cross-talk effects in images reconstructed from measurements at wavelengths selected using our method against those from measurements at wavelengths selected using Corlu et al.'s method. ${ }^{16}$ The simulated medium consisted of a slab with 59,202 nodes, resulting in 38,572 elements, and dimensions $85 \times 85$ $\times 30 \mathrm{~mm}$. An array with eight detectors and eight sources (described previously by Correia et al. ${ }^{39}$ ) is centrally placed at $z=0 \mathrm{~mm}$. However, here only source-detector separations from 14 to $41 \mathrm{~mm}$ were considered. TOAST was used to generate $\mathrm{CW}$ model data at each wavelength, to which $5 \%$ random Gaussian noise was added. The background had brain optical properties, which were described in Section 3.1.1. Hence, the deoxy-hemoglobin concentration $[\mathrm{HHb}]$ is $24 \mu \mathrm{M}$, the oxy-hemoglobin concentration $\left[\mathrm{HbO}_{2}\right]$ is $56 \mu \mathrm{M}$, and the scattering amplitude $a$ is $32 \mathrm{~mm}^{-1} \mathrm{~nm}^{-1}$. Three perturbations with dimensions $10.6 \times 10.6 \times 10.6 \mathrm{~mm}$ were placed within the medium, as shown in Fig. 2. The center of each perturbation was at $11.7 \mathrm{~mm}$ below the imaging surface. A single parameter was changed for each perturbation, which consisted of either an increase in $[\mathrm{HHb}]$ of $1 \mu \mathrm{M}$, in $\left[\mathrm{HbO}_{2}\right]$ of $4 \mu \mathrm{M}$, or in $a$ of $1 \mathrm{~mm}^{-1} \mathrm{~nm}^{-1}$. These perturbations are sufficiently small to ensure that the concentrations and scattering amplitude can be retrieved by linear reconstruction, and are roughly consistent with the magnitude of cerebral hemodynamic changes. Images were reconstructed using the multispectral method on a $24 \times 24 \times 9$ regular pixel basis.

\section{Results}

\subsection{Optimal Wavelengths}

For simplicity, the absorption and diffusion sensitivity profiles are dealt with separately. The first results are shown in Fig. 3 for the homogeneous model, considering absorption sensitivity profiles only and three wavelength sets. Note that the SSD 


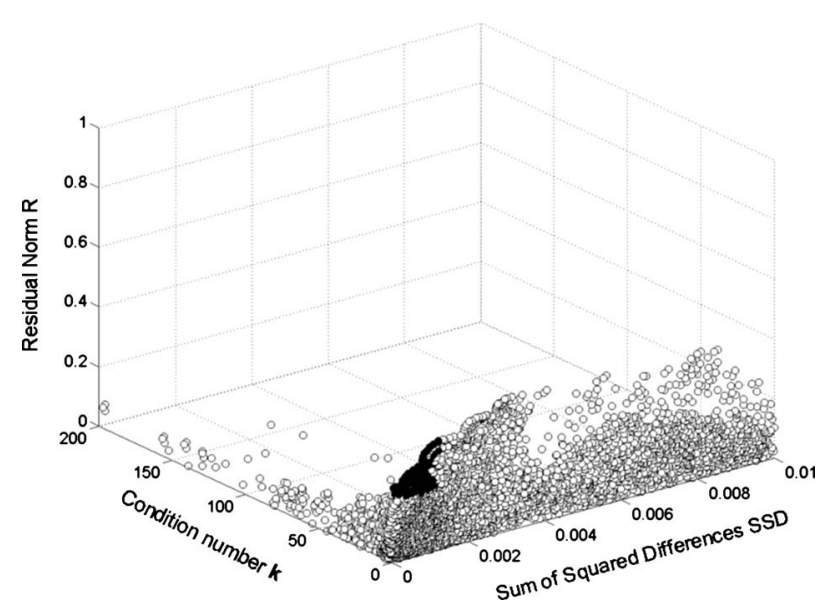

Fig. 3 Plot of the sum of squared differences SSD (in millimeters), condition number $\mathbf{k}$ and residual norm $R$, where each point represents a set of three wavelengths.

values in Fig. 3 represent the average for each wavelength set. Because of the large number of points, further analysis of the wavelength sets was limited to those for which $R \geqslant 0.2, \mathbf{k}$ $\leqslant 100$, and (because the SSD for two similar PMDFs is $\sim 0.001$ ) $\mathrm{SSD} \leqslant 0.002$ (dark circles in Fig. 3). Each criterion has a different order of magnitude; hence, the results contained in this new range were normalized. The distance between the criteria values of a certain set of wavelengths in this range and the point $(\operatorname{SSD}, \mathbf{k}, R)=(0,0,1)$ was also calculated, which is the point with the smallest SSD, smallest condition number criteria, and highest residual. The wavelength distribution of the ten sets of three wavelengths with the shortest distance are displayed in the histogram in Fig. 4. Histograms are obtained by counting the number of times a certain wavelength occurs, which is done separately for the three wavelengths, and normalized to the most frequently occurring wavelength. For comparison, Fig. 5 shows the histogram for the ten wavelength sets within the limit $R \geqslant 0.2$ and

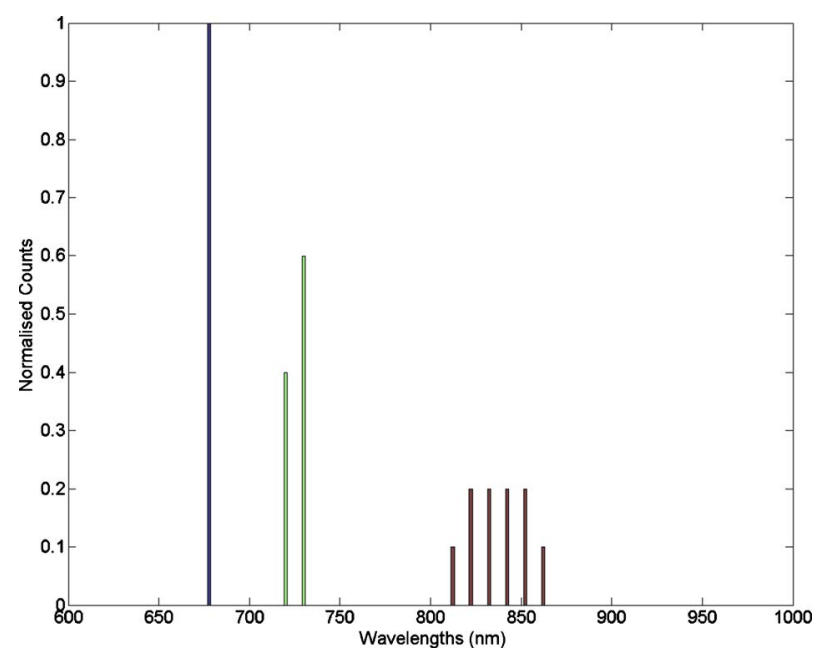

Fig. 4 Histogram of the optimal wavelength distributions that satisfy the three wavelength selection conditions, for the homogeneous model, three wavelengths, and two chromophores $\left(\mathrm{HbO}_{2}\right.$ and $\left.\mathrm{HHb}\right)$.

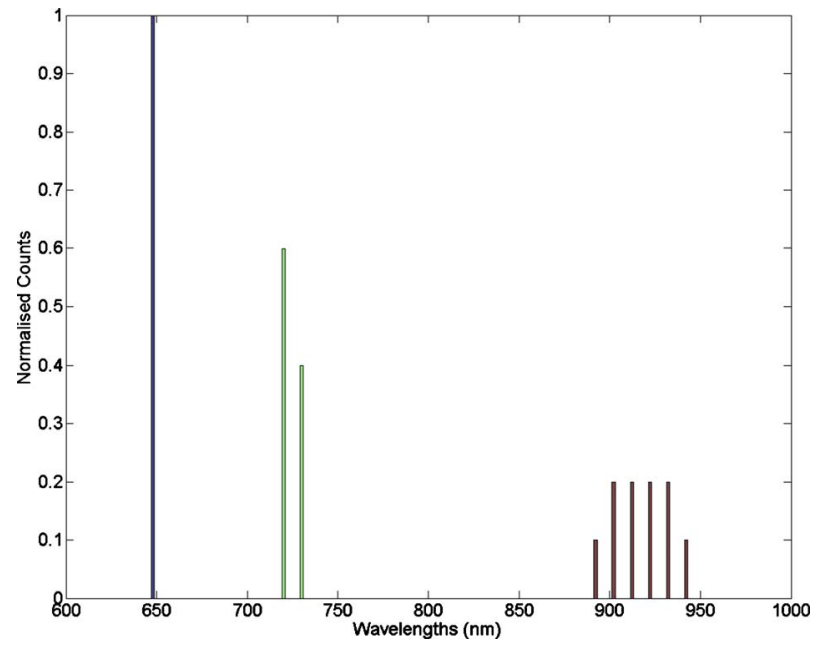

Fig. 5 Histogram of the optimal wavelength distributions that satisfy the high residual and low condition number criteria, for the homogeneous model, three wavelengths, and two chromophores $\left(\mathrm{HbO}_{2}\right.$ and $\mathrm{HHb})$.

$\mathbf{k} \leqslant 100$, whose distance is closest to the point $(\mathbf{k}, R)=(0,1)$. The means and standard deviations $\sigma$ of each wavelength distribution were calculated in order to find the optimal wavelengths and measure their spread. A 5-nm uncertainty error is combined with $\sigma\left(\sigma_{\text {total }}=\sqrt{\sigma^{2}+5^{2}}\right)$, due to the bin size. Using the three-wavelength selection criteria, the wavelengths are $680 \pm 5,726 \pm 7$, and $835 \pm 17 \mathrm{~nm}$. When only the high residual and low condition number criteria are used the optimal wavelengths are $650 \pm 5,724 \pm 7$, and $915 \pm 17 \mathrm{~nm}$. The results suggest that when the new PMDF overlap condition is included the smallest wavelength becomes larger, and the largest wavelength becomes smaller.

The optimal four wavelengths that satisfy the three criteria are $680 \pm 5,715 \pm 14,733 \pm 7$, and $828 \pm 9 \mathrm{~nm}$. Adding a fourth wavelength broadened the central wavelength range and shifted the highest wavelength to a smaller value. The wavelength set obtained without including the third condition is $650 \pm 5,715 \pm 7,727 \pm 7$, and $919 \pm 13 \mathrm{~nm}$. The differences between these results and those presented by Corlu et al. ${ }^{16}$ are inevitably due to differences in the wavelength range and spacing, chromophore extinction coefficients, and criteria limits imposed.

The SSD for diffusion PMDFs is always slightly smaller than for absorption PMDFs. This is probably because scattering does not have a strong wavelength dependence. Thus, the optimal wavelength sets found using the absorption SSDs also return small diffusion SSDs.

The histograms for the three-layer adult head model for three and four wavelengths are shown in Figs. 6 and 7, respectively. For the three wavelength distributions, the optimal wavelength values are $680 \pm 5,725 \pm 10$, and $876 \pm 12 \mathrm{~nm}$, and for four wavelengths these values are $685 \pm 7,719 \pm 9$, $731 \pm 8$, and $873 \pm 9 \mathrm{~nm}$. The main difference between the optimal wavelengths found for the homogeneous model and the three-layer model occurs for the largest wavelength, which becomes larger for the latter. The other wavelengths are approximately the same and, similar to the homogeneous case, adding a fourth wavelength resulted in the merger of the two 


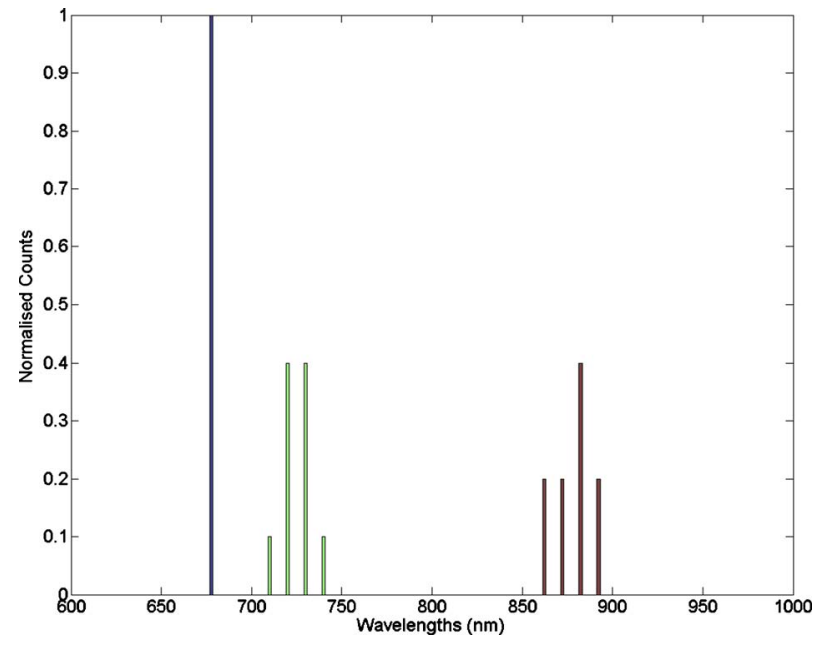

Fig. 6 Histogram of the optimal wavelength distributions that satisfy the three wavelength selection conditions, for the three-layer model, three wavelengths and two chromophores $\left(\mathrm{HbO}_{2}\right.$ and $\left.\mathrm{HHb}\right)$.

central wavelengths (i.e., the mean value is approximately the same but the range is larger). Also for this model, the diffusion SSDs are smaller than the corresponding values for absorption; hence, the previous results also apply for diffusionsensitivity profiles.

The wavelength distributions obtained using our method were similar when the scattering power $b$ was varied from 0 to 2.8 , whereas for the two-criteria method similar wavelength distributions were obtained for a range of $b$ from 0 to 1.2.

\subsubsection{Two wavelengths}

It is common to assume that scattering is homogeneous and constant over time. This assumption is convenient when data are acquired at only two wavelengths, enabling solutions to be obtained if only two chromophores are considered. The following analysis is aimed at identifying the two optimal wave-

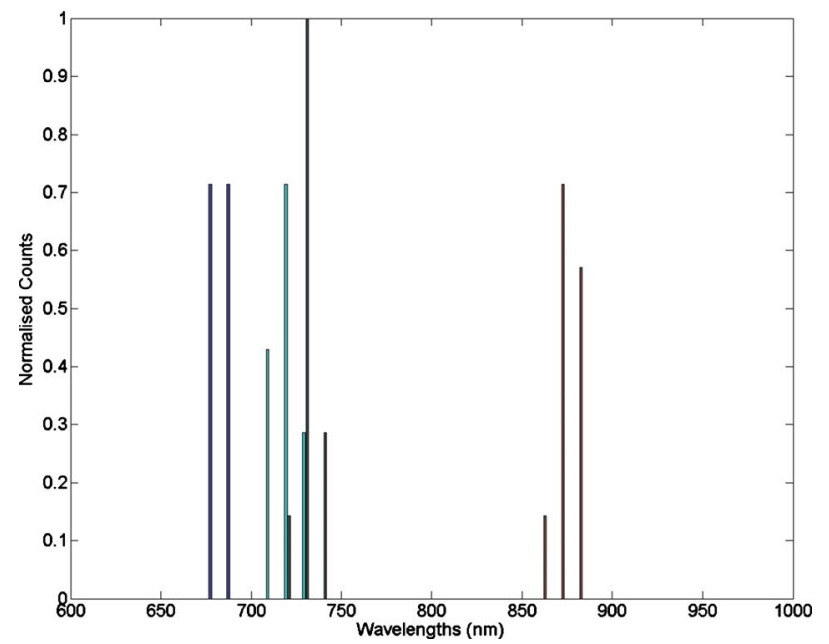

Fig. 7 Histogram of the optimal wavelength distributions that satisfy the three-wavelength selection conditions, for the three model, four wavelengths, and two chromophores $\left(\mathrm{HbO}_{2}\right.$ and $\left.\mathrm{HHb}\right)$. lengths that give good separation between chromophores and good spatial overlap between sensitivity profiles.

The solution is nonunique when only two wavelengths are used. Although the SSD and the condition numbers $\mathbf{k}$ can be very small, the residual norm $R$ is never larger than $1 \times 10^{-7}$, which confirms that one cannot separate absorption from scattering using only two measurement wavelengths.

Chromophore concentrations can be distinguished if the condition number $\mathbf{k}$ is small. The wavelengths whose distance is shortest to the point with the smallest condition number cluster at $710 \pm 16$ and $973 \pm 9 \mathrm{~nm}$. On the other hand, the wavelengths with shortest distance to the point $(\mathrm{SSD}, \mathbf{k})$ $=(0,0)$ cluster at $694 \pm 7$ and $883 \pm 23 \mathrm{~nm}$. The inclusion of the PMDF overlap condition has a clear effect on the largest wavelength.

For the three-layer model, the wavelength pair with the shortest distance to the point $(\operatorname{SSD}, \mathbf{k})=(0,0)$ is $704 \pm 7$ and $887 \pm 12 \mathrm{~nm}$, which is very similar to the optimal wavelength set found for the homogeneous case.

The influence of $\mu_{a}$ on the absorption and diffusion PMDFs is identical; high absorption coefficients lead to narrower PMDFs. A similar tendency is observed when $\mu_{s}^{\prime}$ has a high value for the absorption PMDFs, although the diffusion PMDFs become broader. Nevertheless, the SSD for both absorption and diffusion PMDFs is similar. The wavelength sets obtained using the diffusion PMDFs are identical to those found using the absorption PMDFs. The largest SSD occurs for wavelengths paired with $970 \mathrm{~nm}$, which corresponds to the absorption peak of water. As mentioned previously, the sum of squared differences SSD for diffusion is slightly smaller than for absorption.

\subsection{PMDF Comparison}

\subsubsection{Volume}

The relative volume was calculated for the absorption PMDFs $\left(\mathrm{PMDF}_{\mu_{a}}\right)$ and diffusion PMDFs $\left(\mathrm{PMDF}_{\kappa}\right)$, for both head models. Figure 8 shows the relative volumes for the different wavelengths and the value of $N_{650}$ for each case. The volume of the $\mathrm{PMDF}_{\mu_{a}}$ is larger than that of the $\mathrm{PMDF}_{\kappa}$. For the homogeneous model, the PMDF $\mu_{a}$ volume resembles an inverted $\mu_{a}$ spectrum of brain tissue, because for large $\mu_{a}$ the PMDF volume becomes smaller and for small $\mu_{a}$ the volume becomes larger. For the $\mathrm{PMDF}_{\kappa}$, the volume decreases as expected, given that the volume decreases with decreasing $\mu_{s}^{\prime}$. However, the volume for $\mathrm{PMDF}_{\kappa}$ does not vary as much as for $\mathrm{PMDF}_{\mu_{a}}$. The relative volumes for the layered model are similar to those obtained for the homogeneous case.

\subsubsection{FWHM}

Figure 9 shows the difference between the depth FWHMs and that corresponding to the wavelength $650 \mathrm{~nm}, \mathrm{FWHM}_{650}$. In general, the difference is $<0.6 \mathrm{~mm}$, which indicates that the spread of regions of higher sensitivity does not vary much with wavelength. The FWHM differences are smaller for $\mathrm{PMDF}_{\kappa}$, in particular for the homogeneous model, which shows a relatively flat spectrum for wavelengths of $<970 \mathrm{~nm}$. 


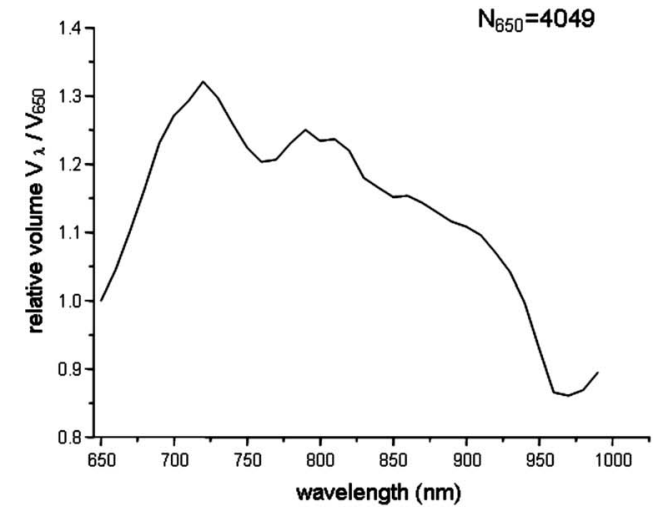

(a)

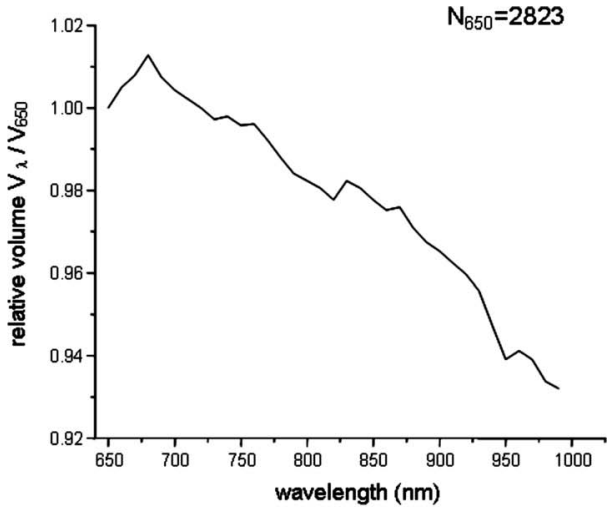

(b)

Fig. 8 Relative volume of (a) the $\mathrm{PMDF}_{\mu_{a}}$ and (b) $\mathrm{PMDF}_{\kappa}$ for the homogeneous head model.

\subsection{3 $P D$}

$\mathrm{PD}$ is larger for the $\mathrm{PMDF}_{\mu_{a}}$, as shown in Fig. 10. The graphs show the differences with respect to the PMDF PD at $650 \mathrm{~nm}, \mathrm{PD}_{650}$. For the homogeneous model, the maximum depth is $15.6 \mathrm{~mm}$ and occurs for a wavelength of $720 \mathrm{~nm}$. The lowest PD is $13.2 \mathrm{~mm}$ and occurs for a wavelength of $970 \mathrm{~nm}$. This means that the maximum PD difference is $2.4 \mathrm{~mm}$. For the layered model, the maximum penetration depth is $14.4 \mathrm{~mm}$ for a wavelength of $720 \mathrm{~nm}$. For a wavelength of $970 \mathrm{~nm}$, the PD attains a minimum value of
$12.0 \mathrm{~mm}$. The largest difference between PD is $2.4 \mathrm{~mm}$. As a consequence of the voxel size, which only allows discrete spatial steps, the relative PD spectra exhibit a steplike behavior.

The $\mathrm{PMDF}_{\kappa}$ exhibits a small variation in the PD for the different wavelengths (Fig. 10). For the homogeneous model, the combination of low $\mu_{a}$ and high $\mu_{s}^{\prime}$ for wavelengths from 670 to $840 \mathrm{~nm}$ results in a broader $\mathrm{PMDF}_{\kappa}$ with higher PD. The maximum PD is $12.6 \mathrm{~mm}$, and the largest difference between PD is $0.6 \mathrm{~mm}$. The $\mu_{s}^{\prime}$ for skull decays faster than for

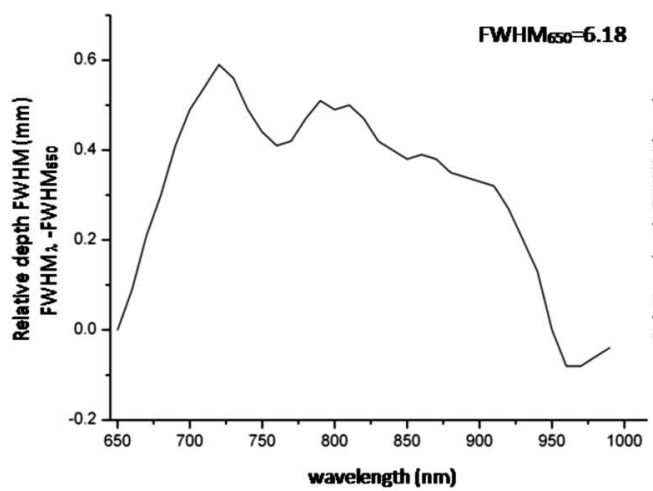

(a)

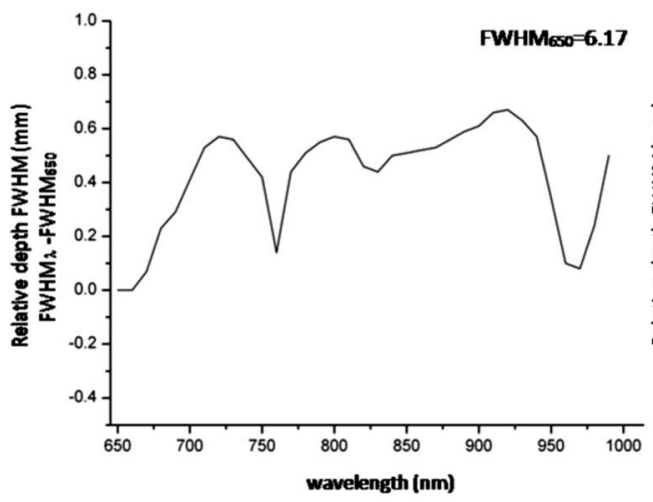

(c)

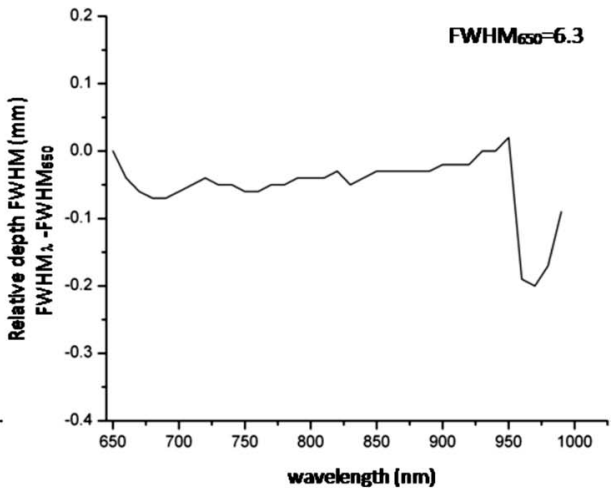

(b)

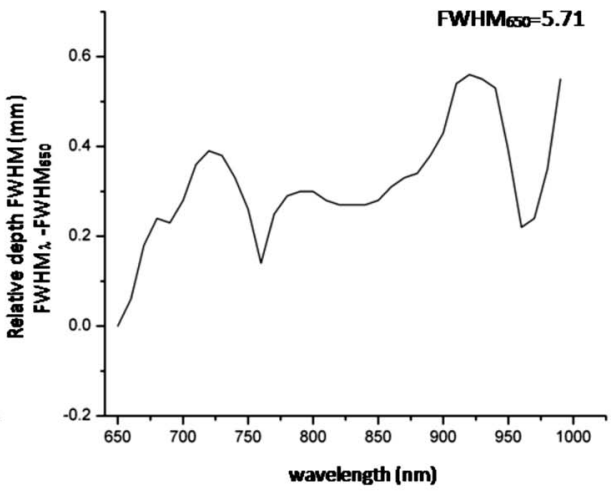

(d)

Fig. 9 FWHM for the depth direction of the (a, c) $\mathrm{PMDF}_{\mu_{\mathrm{a}}}$ and (b, d) $\mathrm{PMDF}_{\kappa}$ profiles for the homogeneous $(\mathrm{a}, \mathrm{b})$ head model and (c, d) layered model. 


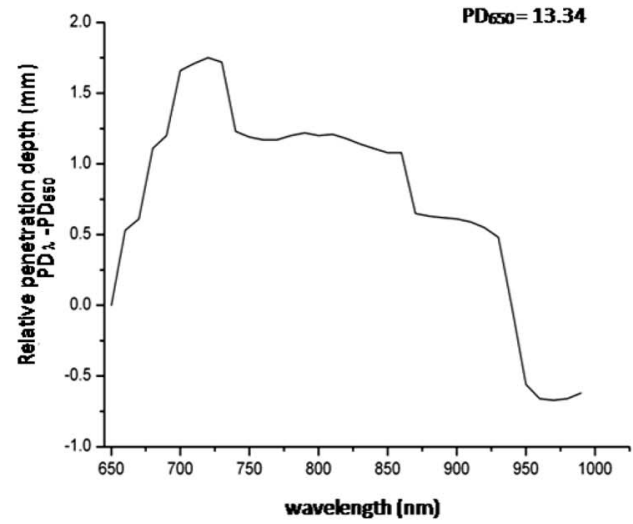

(a)

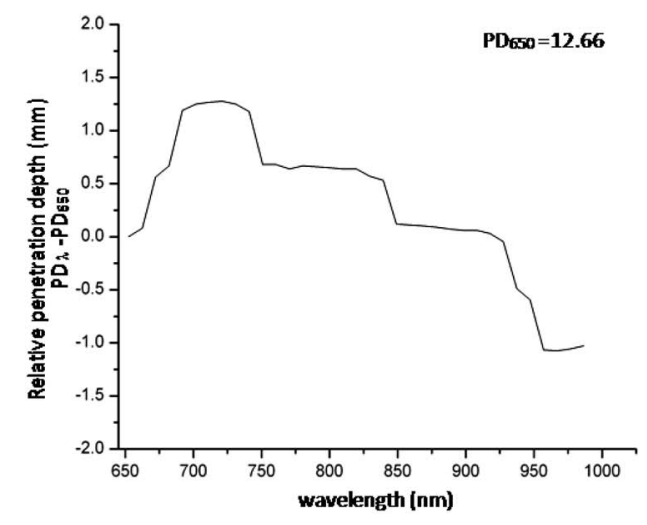

(c)

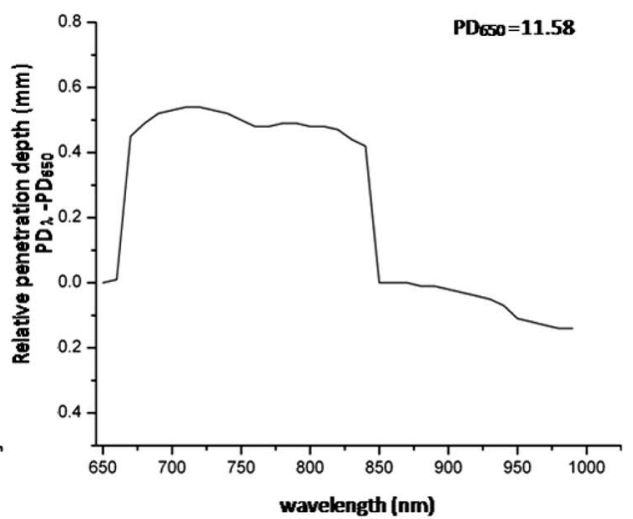

(b)

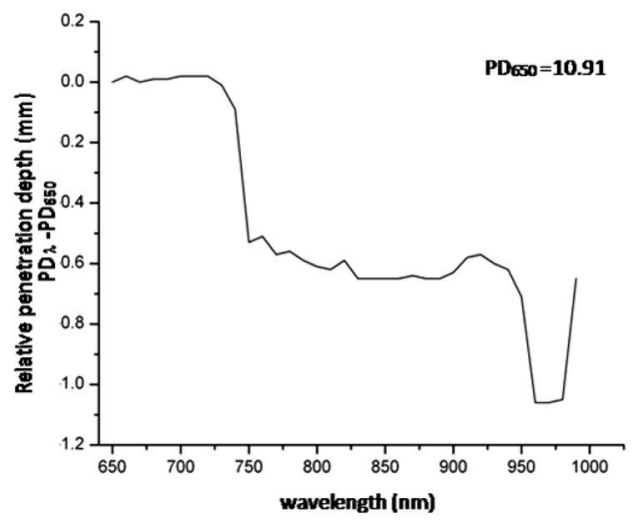

(d)

Fig. 10 Penetration depth of the (a, c) $\mathrm{PMDF}_{\mu_{\mathrm{a}}}$ and (b, d) $\mathrm{PMDF}_{\kappa}$ profiles for the homogeneous (a, b) head model and (c, d) layered model.

brain tissue, and at wavelengths of $>750 \mathrm{~nm}$ their $\mu_{s}^{\prime}$ values diverge significantly. When the external layers have lower $\mu_{s}^{\prime}$ and $\mu_{a}$ than the inner layer, the sensitivity profiles shift toward the surface. The high absorption of the brain tissue at $970 \mathrm{~nm}$ reduces the interrogation depth to $9 \mathrm{~mm}$, compared to the maximum at $11.4 \mathrm{~mm}$.

\subsection{Image Reconstruction}

Images were reconstructed from simulated measurements at wavelengths selected using our new proposed method, and from measurements at wavelengths selected using the method of Corlu et al. ${ }^{16}$ Figure 11 shows the $x y$ cross-sectional images at $z=11.7 \mathrm{~mm}$ [Fig. 11(a)] and $z=8.4 \mathrm{~mm}$ [Fig. 11(b)], using measurement wavelengths at 680,720 , and $830 \mathrm{~nm}$, simultaneously. Figure 12 shows the $x y$ cross-sectional images at $z=11.7 \mathrm{~mm}$ [Fig. 12(a)] and $z=8.4 \mathrm{~mm}$ [Fig. 12(b)], using measurement wavelengths at 650,720 , and $910 \mathrm{~nm}$, simultaneously. Note that the color scales are not the same to enhance the differences between the images. For both sets of wavelengths, the images exhibited cross-talk between chromophores and also scattering. Cross-talk is particularly significant between oxy- and deoxy-hemoglobin concentrations in the deoxy-hemoglobin reconstruction and more evident in the images reconstructed using the wavelengths selected by our method. However, the deoxy-hemoglobin perturbation appears closer to the real position in the images reconstructed from wavelengths selected by our method than in the images obtained from wavelengths selected using the two-condition criteria. The $\left[\mathrm{HbO}_{2}\right]$ is underestimated in all the cases. The $[\mathrm{HHb}]$ is overestimated at $z=8.4 \mathrm{~mm}$ and underestimated at $z=11.7 \mathrm{~mm}$. For comparison, images were reconstructed from simulated measurements at two wavelengths that are commonly used, 780 and $850 \mathrm{~nm}$, and a third wavelength located between these two at $820 \mathrm{~nm}$. Figure 13(a) shows the $x y$ cross-sectional images for this wavelengths set at $z$ $=11.7 \mathrm{~mm}$ and Fig. 13(b) the reconstructed images at $z$ $=8.4 \mathrm{~mm}$. Cross-talk is clearly visible in the oxy-hemoglobin concentration and scattering amplitude images. The calculated concentration and scattering amplitude values are underestimated, except for $[\mathrm{HHb}]$ calculated at $z=8.4 \mathrm{~mm}$. However, the $[\mathrm{HHb}]$ does not appear in the images in the correct position.

The same simulation study was performed using four wavelengths, and the corresponding $x y$ cross-sectional images at $z=11.7 \mathrm{~mm}$ and $z=8.4 \mathrm{~mm}$ obtained using the wavelengths found using our method are shown in Figs. 14(a) and 14(b), respectively. Because of the limited sensitivity of the measurement to scattering to regions deeper than $12 \mathrm{~mm}$ below the surface, the reconstructions were found to have less cross-talk at $z=8.4 \mathrm{~mm}$ than at the true target depth $z$ $=11.7 \mathrm{~mm}$. Figure 15 shows the $x y$ cross-sectional images at $z=11.7 \mathrm{~mm}$ [Fig. 15(a)] and $z=8.4 \mathrm{~mm}$ [Fig. 15(b)], using the wavelengths selected by the two-criteria method. Reconstructions at $z=8.4 \mathrm{~mm}$ for both sets of wavelengths were similar and showed very small cross-talk. Nevertheless, the scattering amplitude and deoxy-hemoglobin concentration 
Correia, Gibson, and Hebden: Identification of the optimal wavelengths for optical topography: a photon measurement...

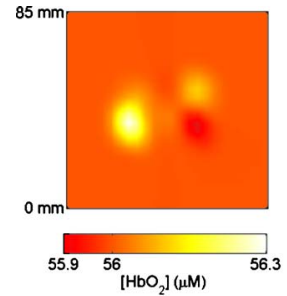

(a)

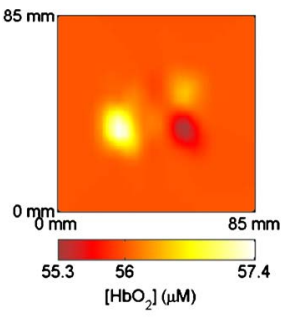

(d)

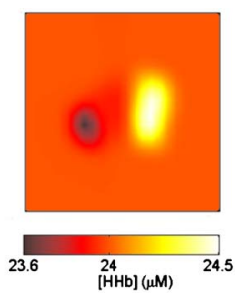

(b)

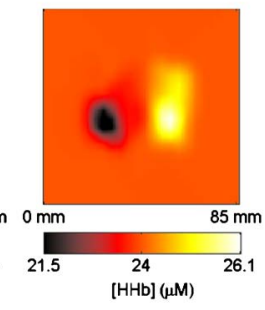

(e)

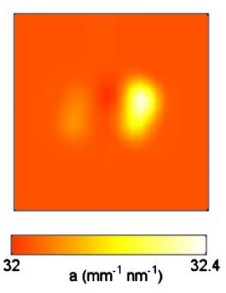

(c)

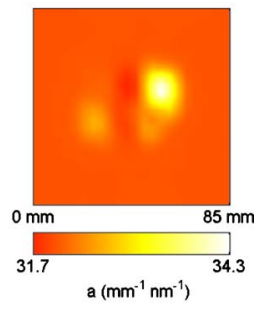

(f)
Fig. 11 Reconstructed images at $(a-c) \quad z=11.7 \mathrm{~mm}$ and $(d-f) \quad z$ $=8.4 \mathrm{~mm}$ of $(\mathrm{a}, \mathrm{d})$ oxy-hemoglobin, $(\mathrm{b}, \mathrm{e})$ deoxy-hemoglobin, and (c, f) scattering amplitude from measurements at wavelengths 680, 720, and $830 \mathrm{~nm}$, for the homogeneous model.

calculated at $z=8.4 \mathrm{~mm}$ were overestimated. The slightly better reconstructions at $z=11.7 \mathrm{~mm}$ obtained using our wavelength set could be due to the higher sensitivity of these wavelengths to the depth at which the targets are located. Furthermore, even though the sensitivity to scattering is quite limited in deeper regions, the PD of the $\mathrm{PMDF}_{\kappa}$ has its maximum at these wavelengths. For comparison, images were reconstructed using the wavelengths $750,780,820$, and $850 \mathrm{~nm}$. Figure 16(a) shows the $x y$ cross-sectional images at $z=11.7 \mathrm{~mm}$, and Fig. 16(b) shows the images at $z=8.4 \mathrm{~mm}$,

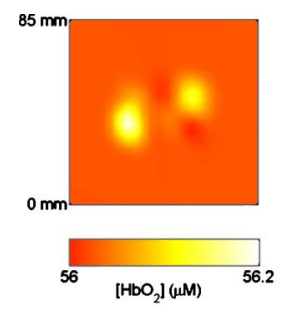

(a)

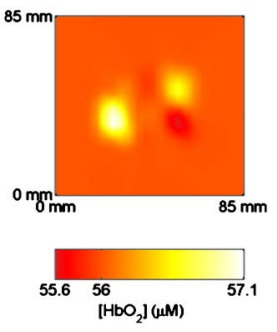

(d)

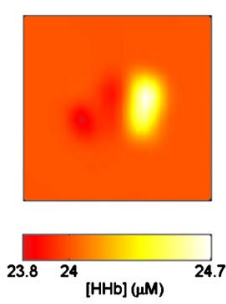

(b)

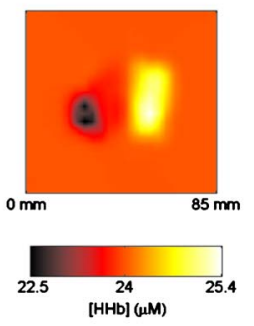

(e)

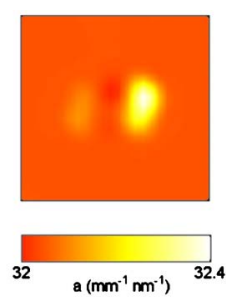

(c)

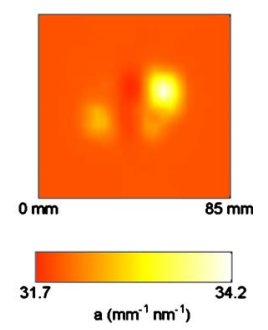

(f)
Fig. 12 Reconstructed images at $(a-c) \quad z=11.7 \mathrm{~mm}$ and $(d-f) \quad z$ $=8.4 \mathrm{~mm}$ of $(\mathrm{a}, \mathrm{d})$ oxy-hemoglobin, (b, e) deoxy-hemoglobin, and (c, f) scattering amplitude from measurements at wavelengths 650,720 , and $910 \mathrm{~nm}$, for the homogeneous model.

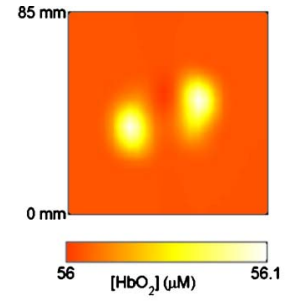

(a)

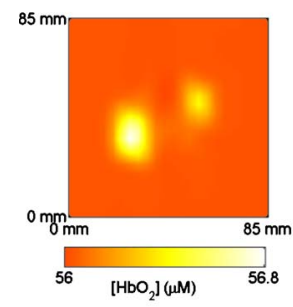

(d)

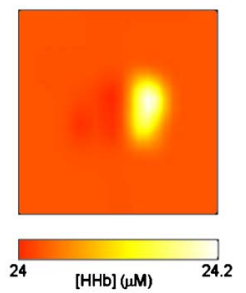

(b)

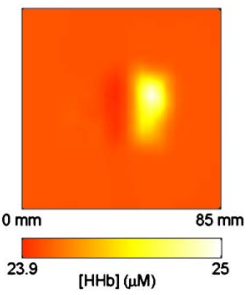

(e)

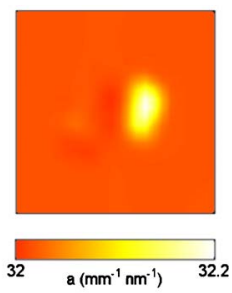

(c)

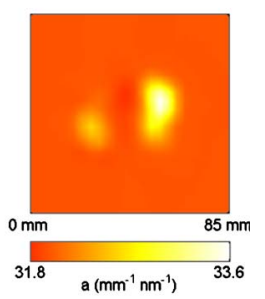

(f)
Fig. 13 Reconstructed images at $(\mathrm{a}-\mathrm{c}) \quad z=11.7 \mathrm{~mm}$ and $(\mathrm{d}-\mathrm{f}) \quad z$ $=8.4 \mathrm{~mm}$ of $(\mathrm{a}, \mathrm{d})$ oxy-hemoglobin, $(\mathrm{b}, \mathrm{e})$ deoxy-hemoglobin, and (c, f) scattering amplitude from measurements at wavelengths 780, 820, and $850 \mathrm{~nm}$, for the homogeneous model.

obtained using the latter measurement set. Cross-talk is visible in the images; the calculated chromophore concentrations and scattering amplitude are underestimated; and the deoxyhemoglobin perturbation appears misplaced in the image.

The simulation results indicate that four measurement wavelengths are required, in order to reconstruct images with negligible cross-talk, from reflection measurements and using linear multispectral reconstruction.

For the three-layer adult head model presented here, the sensitivity to scattering perturbation occurring in the brain

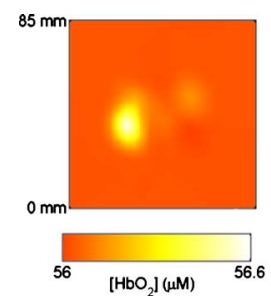

(a)

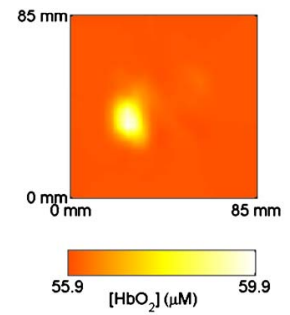

(d)

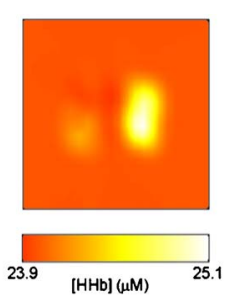

(b)

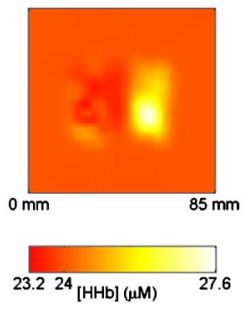

(e)

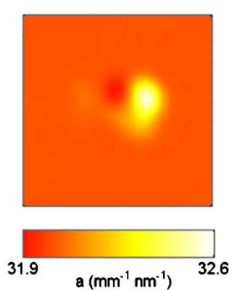

(c)

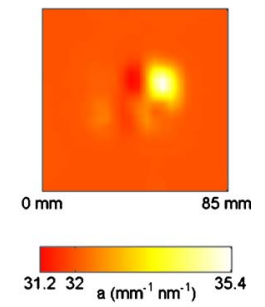

(f)
Fig. 14 Reconstructed images at $(\mathrm{a}-\mathrm{c}) \quad z=11.7 \mathrm{~mm}$ and $(\mathrm{d}-\mathrm{f}) \quad z$ $=8.4 \mathrm{~mm}$ of $(\mathrm{a}, \mathrm{d})$ oxy-hemoglobin, (b, e) deoxy-hemoglobin, and (c, f) scattering amplitude from measurements at wavelengths $680 \mathrm{~nm}$, 710,730 , and $830 \mathrm{~nm}$, for the homogeneous model. 
Correia, Gibson, and Hebden: Identification of the optimal wavelengths for optical topography: a photon measurement...

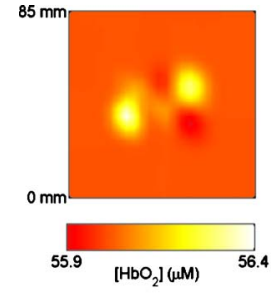

(a)

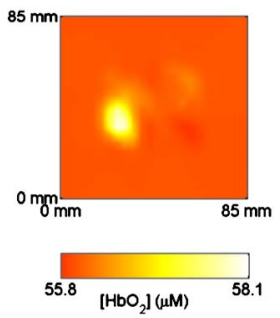

(d)

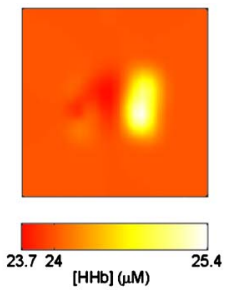

(b)

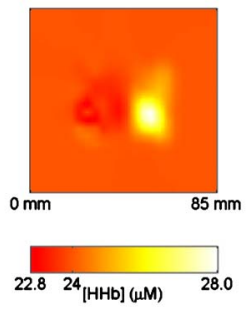

(e)

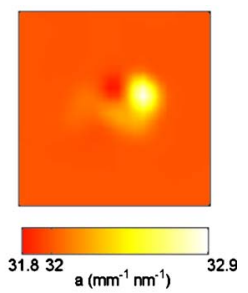

(c)

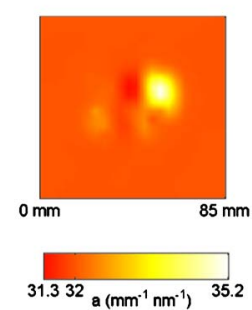

(f)
Fig. 15 Reconstructed images at $(\mathrm{a}-\mathrm{c}) \quad z=11.7 \mathrm{~mm}$ and $(\mathrm{d}-\mathrm{f}) \quad z$ $=8.4 \mathrm{~mm}$ of $(\mathrm{a}, \mathrm{d})$ oxy-hemoglobin, $(\mathrm{b}, \mathrm{e})$ deoxy-hemoglobin, and $(\mathrm{c}$, f) scattering amplitude from measurements at wavelengths 650,710 , 730, and $910 \mathrm{~nm}$, for the homogeneous model.

region is very low; hence, it is not possible to separate the two chromophores and scattering amplitude simultaneously.

Finally, simulations were performed using the same medium as before, but without the scattering perturbation and for two wavelengths only. Figure 17 shows the reconstructions at $z=11.7 \mathrm{~mm}$ and $z=8.4 \mathrm{~mm}$ obtained from measurements at wavelengths found using our method (690 and $880 \mathrm{~nm}$ ). The images show a good separation between chromophores, but at $z=8.4 \mathrm{~mm}$, the calculated chromophore concentrations are overestimated and, at $z=11.7 \mathrm{~mm}$, are slightly underestimated. The images reconstructed from measurements at wavelengths found using the two criteria method (710 and

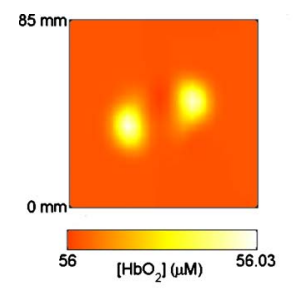

(a)

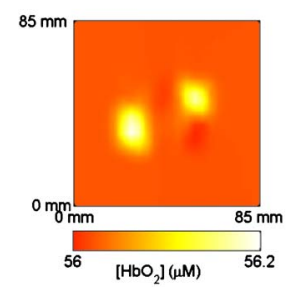

(d)

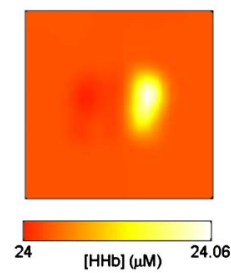

(b)

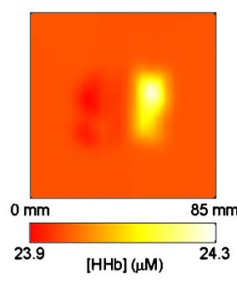

(e)

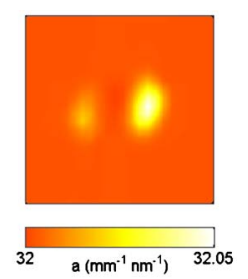

(c)

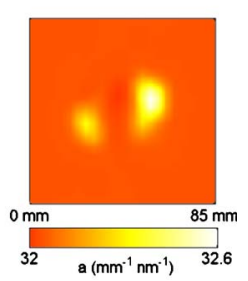

(f)
Fig. 16 Reconstructed images at $(a-c) \quad z=11.7 \mathrm{~mm}$ and $(\mathrm{d}-\mathrm{f}) \quad z$ $=8.4 \mathrm{~mm}$ of $(\mathrm{a}, \mathrm{d})$ oxy-hemoglobin, (b, e) deoxy-hemoglobin, and (c, f) scattering amplitude from measurements at wavelengths 750, 780, 820 , and $850 \mathrm{~nm}$, for the homogeneous model.

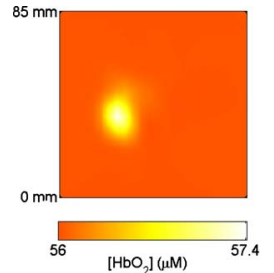

(a)

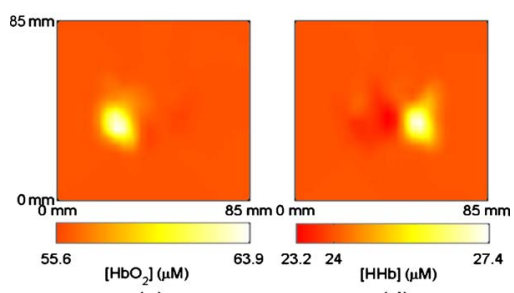

(c)

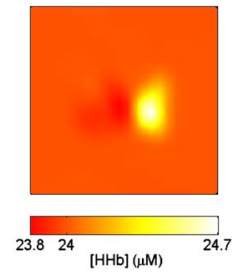

(b)

(d)
Fig. 17 Reconstructed images at $(\mathrm{a}, \mathrm{b}) \mathrm{z}=11.7 \mathrm{~mm}$ and $(\mathrm{c}, \mathrm{d}) \mathrm{z}$ $=8.4 \mathrm{~mm}$ of $(\mathrm{a}, \mathrm{c})$ oxy-hemoglobin, and (b, d) deoxy-hemoglobin from measurements at wavelengths 690 and $880 \mathrm{~nm}$, for the homogeneous model.

$970 \mathrm{~nm}$ ) exhibit a small amount of cross-talk (Fig. 18). The presence of cross-talk could be due to sensitivity differences between the two wavelengths. At $970 \mathrm{~nm}$, only the top of the target is interrogated, whereas the light at $710 \mathrm{~nm}$ is likely to travel through the whole target volume.

All the simulations were repeated for up to three times larger concentration and scattering amplitude changes, and the results showed a similar tendency.

In applications of optical topography, the true optical properties of the medium are usually unknown; hence, the sensitivity matrix is usually calculated using an estimate of the average optical properties, and subsequently used to reconstruct images from measurements at different wavelengths. Given that the optical properties of the medium are wavelength dependent, the reconstructed images are affected by using sensitivity matrices generated for optical properties dif-

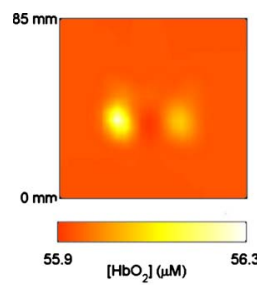

(a)

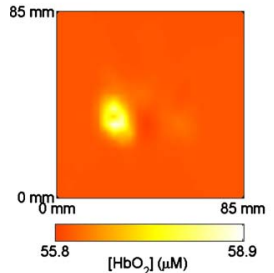

(c)

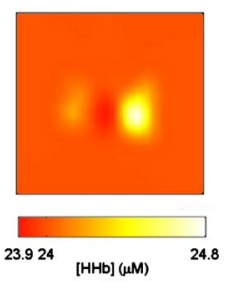

(b)

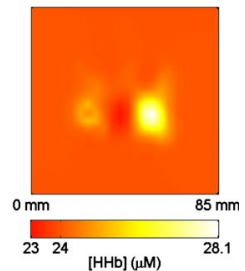

(d)
Fig. 18 Reconstructed images at (a, b) $z=11.7 \mathrm{~mm}$ and (c, d) $z$ $=8.4 \mathrm{~mm}$ of $(\mathrm{a}, \mathrm{c})$ oxy-hemoglobin, and $(\mathrm{b}, \mathrm{d})$ deoxy-hemoglobin from measurements at wavelengths 710 and $970 \mathrm{~nm}$, for the homogeneous model. 
ferent from the true properties. If the estimate of the optical properties is significantly different from the true values, then the absorption perturbation is likely to appear misplaced in the image, particularly in the $z$ direction.

\section{Discussion}

A method of determining the optimum wavelengths for optical topography is presented. This method is based on the multispectral method and is an extension of Corlu's wavelength selection method, ${ }^{16}$ where a third criterion is introduced that minimizes the differences between interrogated volumes at different wavelengths. Including the sensitivity profiles similarity criterion in the selection of the optimal wavelengths has a clear effect on the resultant wavelength sets. The insertion of two superficial layers in the adult head model had an influence on the results, in particular, for the largest wavelength mean value, which shows that selection depends on the accuracy of the head model. It is possible to separate $\left[\mathrm{HbO}_{2}\right]$ and $[\mathrm{HHb}]$ from measurements performed at two wavelengths. However, one cannot distinguish between absorption and scattering. When the sensitivity profiles similarity condition is included in the wavelength selection method, the largest wavelength mean value changes in comparison to that obtained using the condition $\mathbf{k}$ alone.

The main characteristics of the optically interrogated volumes (volume, FWHM, and PD) were quantified, in order to determine how measurements at different NIR wavelengths may be combined to provide information about the volume. Particular attention is given to the wavelengths selected using our wavelength selection method and Corlu's method. ${ }^{16}$

If we consider the thickness of the head superficial layers to be $\sim 11 \mathrm{~mm}$ and the gray matter to be $4 \mathrm{~mm}$, then in theory for the homogeneous model, the lowest wavelength selected using the two-criteria method penetrates $2.8 \mathrm{~mm}$ into the gray matter, whereas the midrange wavelengths penetrate $4.6 \mathrm{~mm}$, which means that some light could reach the white matter. Therefore, the information contained in the measurements at these wavelengths could be significantly different. Including our PMDF overlap condition in the selection of the optimal wavelengths reduces the difference in the PD from 1.8 to $0.6 \mathrm{~mm}$. The first and last wavelengths, with mean values 680 and $830 \mathrm{~nm}$, respectively, penetrate $4 \mathrm{~mm}$ into the brain tissue. This value is closer to that obtained for the central wavelengths, which is $4.6 \mathrm{~mm}$. The volumes interrogated are also larger and more similar for the wavelengths selected using our method. For the $\mathrm{PMDF}_{\kappa}$, the changes in the PD are smaller and the maximum PD is only $12.6 \mathrm{~mm}$, hence confined to the gray matter. However, the differences between PD are smaller when our method is used.

For the three-layer adult head model, the discrepancies between the interrogated volumes at different wavelengths are smaller. However, the volumes are more similar for the wavelengths selected using our method than using the twoselection criteria. In terms of PD, for the $\mathrm{PMDF}_{\mu_{a}}$, the variations are smaller than for the homogeneous model. For the central wavelengths of the selected wavelength sets light penetrates $3.5 \mathrm{~mm}$ into the gray matter. For the lowest wavelengths obtained with our method, light penetrates $2.9 \mathrm{~mm}$ into the brain. For the remaining wavelengths, a maximum depth of $2.2 \mathrm{~mm}$ from the brain surface is probed. Although the differences between PD for the different wavelengths are identical for both selection methods, when using our method it optimizes for the wavelength set with higher PD, simultaneously with the best separation between chromophores, absorption, and scattering. The PMDF spread (i.e., the FWHM and volume) is also larger and more similar for our wavelength set. For the $\mathrm{PMDF}_{\kappa}$, the maximum $\mathrm{PD}$ is only $11.4 \mathrm{~mm}$; hence, light hardly reaches the brain surface. The differences $\mathrm{PMDF}_{\kappa}$ are much smaller than for the absorption PMDFs.

From this analysis, it was shown that for the adult human head it can be assumed that the volumes of interrogated tissue at different NIR wavelengths are identical. Hence, measurements performed at different NIR wavelengths provide information from the same brain regions.

This method could be applied to other types of tissue. For example, for measurements of muscle, breast, or neonate brain. For muscle, the highest absorption occurs at $650 \mathrm{~nm}$ and the lowest at $\sim 850 \mathrm{~nm} .{ }^{40}$ For these wavelengths, the PD is 15 and $21.6 \mathrm{~mm}$, respectively. This results in a PD maximum difference of $6.6 \mathrm{~mm}$. For studies of muscle exercise, of the forearm for example, which has a diameter of $\sim 7 \mathrm{~cm}$, a difference of $\sim 7 \mathrm{~mm}$ is quite significant. The influence of the skin and fat layer on the light propagation is likely to increase the PD, and it should depend on the thickness of these layers. For breast tissue, using the optical properties from Cubeddu et al., ${ }^{41}$ the largest differences in the absorption coefficient occur at wavelengths 720 and $970 \mathrm{~nm}$, with a PD of 22 and $16 \mathrm{~mm}$, respectively. Therefore, the maximum PD difference is $6 \mathrm{~mm}$. The main objective of breast diffuse optical imaging is tumor detection. It is generally assumed that a tumor may be distinguished from surrounding healthy tissue on the basis of its apparent blood volume and oxygenation. Overlapping sensitivities are required to provide accurate measures of these parameters and reduce the uncertainty in the measured spatial location of the tumour.

Light propagation in the neonatal brain is different from the adult brain tissue, because the surrounding layers are thinner and the optical properties are different. The $\mu_{s}^{\prime}$ is lower for the neonatal brain tissue. ${ }^{27}$ Because of the high scattering of the neonatal brain, the sensitive regions of the PMDF should be confined to the gray matter. However, the cortical thickness is only $\sim 2.5 \mathrm{~mm}$, meaning that light is likely to penetrate into deeper regions of the white matter. ${ }^{42}$

The PMDFs of other types of measurements, either frequency or time domain, can also be analyzed using our method. For example, the region of higher sensitivity of the mean time or phase PMDF is located deeper in the tissue than that of the CW PMDFs. ${ }^{43}$

It is clear that the adult head model had a large influence on the results. A more complete model of the adult head should include the cerebrospinal fluid (CSF) surrounding the brain tissue. However, the diffusion equation-based model is only valid for high scattering tissues and cannot be applied to the low scattering CSF. Monte Carlo simulations of light transport can be used, but they require long computational times. An alternative method is the hybrid radiosity-diffusion method, where the light propagation in high scattering media is calculated using the diffusion equation and the low scattering region by radiosity theory. ${ }^{44,45}$ 
The inclusion of a CSF layer in our adult head model would not significantly affect our results because the high sensitivity regions of the PMDF would still be confined to superficial regions of the brain and because this layer has very little wavelength dependence. The PD of the light in models with and without CSF is almost the same, for source-detector separations of 30 and $40 \mathrm{~mm}^{31}$

A further consideration is the error associated with the extinction coefficient for the different chromophores. The spectra found in the literature, for certain chromophores, show some variation. Based on Corlu's method ${ }^{16}$ for wavelength optimization, Brendel and Nielsen ${ }^{46}$ added a criterion that evaluates the reconstruction errors due to uncertainty in the extinction coefficients. From simulations and breastlike phantom measurements, they found a set of optimum wavelengths almost independent on the uncertainties in the extinction coefficients spectra. Another potential source of error is the assumed values of the chromophore concentrations for each head layer. This influences the optical properties of the layers, which could possibly have an effect on the spatial sensitivity profiles.

Contrary to previous findings, our simulation results indicate that four measurement wavelengths are required in order to reconstruct images with low cross-talk from reflection measurements and using linear multispectral reconstruction. Images reconstructed from simulated measurements at wavelengths selected using our method exhibit a smaller cross-talk than those reconstructed from measurements selected using the two criteria. Therefore, the use of measurements with overlapping sensitivities slightly improves the image reconstruction.

\section{Acknowledgments}

The work has been supported by a scholarship awarded to T.C. by Fundação para a Ciência e a Tecnologia, Portugal. This work has been supported in part by funding from the ECs seventh framework programme under Grant Agreement No. FP7-HEALTH-2007-201076.

\section{References}

1. A. P. Gibson, T. Austin, N. L. Everdell, M. Schweiger, S. R. Arridge, J. H. Meek, J. S. Wyatt, D. T. Delpy, and J. C. Hebden, "Threedimensional whole-head optical tomography of passive motor evoked responses in the neonate," Neuroimage 30, 521-528 (2006).

2. L. C. Enfield, A. P. Gibson, N. L. Everdell, D. T. Delpy, M. Schweiger, S. R. Arridge, C. Richardson, M. Keshtgar, M. Douek, and J. C. Hebden, "Three-dimensional time-resolved optical mammography of the uncompressed breast," Appl. Opt. 46, 3628-3638 (2007).

3. B. W. Zeff, B. R. White, H. Dehghani, B. L. Schlaggar, and J. P Culver, "Retinotopic mapping of adult human visual cortex with high-density diffuse optical tomography," Proc. Natl. Acad. Sci. U.S.A. 104, 12169-12174 (2007).

4. S. R. Hintz, D. A. Benaron, A. M. Zourabian, D. K. Stevenson, and D. A. Boas, "Bedside functional imaging of the premature infant brain during passive motor activation," J. Perinat. Med. 29, 335-343 (2001).

5. A. Custo, D. A. Boas, D. Tsuzuki, I. Dan, R. Mesquita, B. Fischl, W. E. L. Grimson, and W. Wells, "Anatomical atlas-guided diffuse optical tomography of brain activation," Neuroimage 49, 561-567 (2010).

6. R. Choe, S. D. Konecky, A. Corlu, K. Lee, T. Durduran, D. R. Busch, S. Pathak, B. J. Czerniecki, J. Tchou, D. L. Fraker, A. DeMichele, B. Chance, S. R. Arridge, M. Schweiger, J. P. Culver, M. D. Schnall, M. E. Putt, M. A. Rosen, and A. G. Yodh, "Differentiation of benign and malignant breast tumors by in vivo three-dimensional parallel-plate diffuse optical tomography," J. Biomed. Opt. 14, 024020 (2009).

7. J. C. Hebden, "Advances in optical imaging of the newborn infant brain," Psychophysiology 40, 501-510 (2003).

8. K. Uludağ, J. Steinbrink, A. Villringer, and H. Obriga, "Separability and cross talk: optimizing dual wavelength combinations for nearinfrared spectroscopy of the adult head," Neuroimage 22, 583-589 (2004).

9. Y. Yamashita, A. Maki, and H. Koizumi, "Wavelength dependence of the precision of noninvasive optical measurement of oxy-, deoxy-, and total-hemoglobin concentration," Med. Phys. 28, 1108-1114 (2001).

10. G. Strangman, M. A. Franceschini, and D. A. Boas, "Factors affecting the accuracy of near-infrared spectroscopy concentration calculations for focal changes in oxygenation parameters," Neuroimage $\mathbf{1 8}$ 865-879 (2003).

11. N. Okui and E. Okada, "Wavelength dependence of crosstalk in dualwavelength measurement of oxy- and deoxy-hemoglobin," $J$. Biomed. Opt. 10, 011015 (2005).

12. S. Umeyama and T. Yamada, "A new crosstalk measure of nearinfrared spectroscopy and its application to wavelength combination optimization," J. Biomed. Opt. 14, 034017 (2009).

13. H. Sato, M. Kiguchi, and A. Maki, "Wavelength dependence of effective pathlength factor in noninvasive optical measurements of human brain functions," Jpn. J. Appl. Phys. 45, L361-L363 (2006).

14. S. R. Arridge and W. R. B. Lionheart, "Nonuniqueness in diffusionbased optical tomography," Opt. Lett. 23, 882-884 (1998).

15. A. Corlu, T. Durduran, R. Choe, M. Schweiger, E. M. C. Hillman, S. R. Arridge, and A. G. Yodh, "Uniqueness and wavelength optimization in continuous-wave multispectral diffuse optical tomography," Opt. Lett. 28, 2339-2341 (2003).

16. A. Corlu, R. Choe, T. Durduran, K. Lee, M. Schweiger, S. R. Arridge, E. M. C. Hillman, and A. G. Yodh, "Diffuse optical tomography with spectral constrains and wavelength optimization," Appl. Opt. 44, 2082-2093 (2005).

17. S. R. Arridge, "Optical tomography in medical imaging," Inverse Probl. 15, R41-R93 (1999).

18. M. A. OLeary, D. A. Boas, B. Chance, and A. G. Yodh, "Experimental images of heterogeneous turbid media by frequency domain diffusing photon tomography," Opt. Lett. 20, 4268 (1995).

19. M. Cheney, D. Isaacson, J. C. Newell, S. Simske, and J. C. Goble, "NOSER: an algorithm for solving the inverse conductivity problem," Int. J. Imaging Syst. Technol. 2, 66-75 (1990).

20. A. Adler, T. Dai, and W. R. B. Lionheart, "Temporal image reconstruction in electrical impedance tomography," Physiol. Meas 28, S1-S11 (2007).

21. S. R. Arridge, "Photon measurement density functions. Part1: analytical forms," Appl. Opt. 34, 7395-7409 (1995).

22. S. R. Arridge, J. C. Hebden, M. Schweiger, F. E. W. Schmidt, M. E. Fry, E. M. C. Hillman, H. Dehghani, and D. T. Delpy, "A method for three-dimensional time-resolved optical tomography," Int. J. Imaging Syst. Technol. 11, 2-11 (2000).

23. E. Okada, M. Firbank, M. Schweiger, S. R. Arridge, M. Cope, and D. T. Delpy, "Theoretical and experimental investigation of nearinfrared light propagation in a model of the adult head," Appl. Opt. 36, 21-31 (1997).

24. T. S. Leung, C. E. Elwell, and S. T. Delpy, "Estimation of cerebral oxy- and deoxy-haemoglobin concentration changes in a layered adult head model using near-infrared spectroscopy and multivariate statistical analysis," Phys. Med. Biol. 50, 5783-5798 (2005).

25. D. R. White, E. M. Widdowson, H. Q. Woodard, and J. W. Dickerson, "The composition of body tissues (II). fetus to young adult," $\mathrm{Br}$. J. Radiol. 64, 149-159 (1991).

26. M. Cope, "The application of near infrared spectroscopy to non invasive monitoring of cerebral oxygenation in the newborn infant," $\mathrm{PhD}$ thesis, University College London (1991).

27. P. van der Zee, "Measurement and modelling of the optical properties of human tissue in the near-infrared," $\mathrm{PhD}$ thesis, University College London (1992).

28. I. V. Meglinski and S. J. Matcher, "Quantitative assessment of skin layers absorption and skin reflectance spectra simulation in the visible and near-infrared spectral regions," Physiol. Meas 23, 741-753 (2002).

29. George Zonios, Julie Bykowski, and Nikiforos Kollias, "Skin melanin, hemoglobin, and light scattering properties can be quantitatively 
assessed in vivo using diffuse reflectance spectroscopy," J. Invest. Dermatol. 117, 1452-1457 (2001).

30. S. L. Jacques, "Skin optics," 〈http://omlc.ogi.edu/news/jan98/ skinoptics.html $>$ (1998).

31. E. Okada and D. T. Delpy, "Near-infrared light propagation in an adult head model. I. Modeling of low-level scattering in the cerebrospinal fluid layer," Appl. Opt. 42, 2906-2914 (2003).

32. S. J. Matcher, M. Cope, and D. T. Delpy, "Use of the water absorption spectrum to quantify tissue chromophore concentration changes in near-infrared spectroscopy," Phys. Med. Biol. 39, 177-196 (1994).

33. spectra UCL, 〈http://www.medphys.ucl.ac.uk/research/borl/research/ NIR_topics/spectra/spectra.htm $\rangle,(2005)$.

34. R. L. P. van Veen, H. J. C. M. Sterenborg, A. Pifferi, A. Torricelli, and R. Cubeddu, "Determination of VIS- NIR absorption coefficients of mammalian fat, with time- and spatially resolved diffuse reflectance and transmission spectroscopy," presented at OSA Annual BIOMED Topical Meeting (2004).

35. S. J. Matcher, M. Cope, and D. T. Delpy, "In vivo measurements of the wavelength dependence of tissue-scattering coefficients between 760 and $900 \mathrm{~nm}$ measured with time-resolved spectroscopy," Appl. Opt. 36, 386-396 (1997).

36. M. Firbank, M. Hiraoka, M. Essenpreis, and D. T. Delpy, "Measurement of the optical properties of the skull in the wavelength range 650-950 nm," Phys. Med. Biol. 38, 503-510 (1993).

37. C. R. Simpson, M. Kohl, M. Essenpreis, and M. Cope, "Near-infrared optical properties of ex vivo human skin and subcutaneous tissues measured using the monte carlo inversion technique," Phys. Med. Biol. 43, 2465-2478 (1998).

38. A. N. Bashkatov, E. A. Genina, V. I. Kochubey, and V. V. Tuchin, "Optical properties of human skin, subcutaneous and mucous tissues in the wavelength range from 400 to $2000 \mathrm{~nm}$," J. Phys. D 38, 25432555 (2005).

39. T. Correia, A. Banga, N. L. Everdell, A. P. Gibson, and J. C. Hebden, "A quantitative assessment of the depth sensitivity of an optical topography system using a solid dynamic tissue-phantom," Phys. Med. Biol. 54, 6277-6286 (2009).

40. J. Xia, A. Weaver, D. E. Gerrard, and G. Yao, "Monitoring sarcomere structure changes in whole muscule using diffuse light reflectance," $J$. Biomed. Opt. 11, 040504 (2006).

41. R. Cubeddu, C. D'Andrea, A. Pifferi, P. Taroni, A. Torricelli, and G. Valentini, "Effects of the menstrual cycle on the red and near-infrared optical properties of the human breast," Photochem. Photobiol. 72, 383-391 (2000).

42. Y. Fukui, Y. Ajichi, and E. Okada, "Monte carlo prediction of nearinfrared propagation in realistic adult and neonatal head models," Appl. Opt. 42, 2881-2887 (2003).

43. S. R. Arridge and M. Schweiger, "Photon measurement density functions. Part2: finite element method calculations," Appl. Opt. 34, 8026-8037 (1995).

44. S. R. Arridge, H. Dehghani, Martin Schweiger, and E. Okada, "The finite element model for the propagation of light in scattering media: a direct method for domains with nonscattering regions," Med. Phys. 27, 252-264 (2000).

45. H. Dehghani, S. R. Arridge, Martin Schweiger, and D. T. Delpy, "Optical tomography in the presence of void regions," J. Opt. Soc. Am. 17, 1659-1670 (2000).

46. B. Brendel and T. Nielsen, "Wavelengths optimization in multispectral diffuse optical tomography considering uncertainties in absorption spectra," Proc. SPIE 6629, 66290A (2007). 